\title{
Minimum Energy Demand Locomotion on Space Station
}

\author{
Wing Kwong Chung ${ }^{1,2}$ and Yangsheng $\mathrm{Xu}^{1,2}$ \\ ${ }^{1}$ Department of Mechanical and Automation Engineering, The Chinese University of Hong Kong, Hong Kong \\ ${ }^{2}$ Shenzhen Institute of Advanced Integration Technology, Chinese Academy of Sciences, The Chinese University of Hong Kong, \\ Shenzhen 518067, China \\ Correspondence should be addressed to Wing Kwong Chung; wkchung@mae.cuhk.edu.hk
}

Received 1 June 2012; Accepted 14 December 2012

Academic Editor: Ou Ma

Copyright (C) 2013 W. K. Chung and Y. Xu. This is an open access article distributed under the Creative Commons Attribution License, which permits unrestricted use, distribution, and reproduction in any medium, provided the original work is properly cited.

\begin{abstract}
The energy of a space station is a precious resource, and the minimization of energy consumption of a space manipulator is crucial to maintain its normal functionalities. This paper first presents novel gaits for space manipulators by equipping a new gripping mechanism. With the use of wheels locomotion, lower energy demand gaits can be achieved. With the use of the proposed gaits, we further develop a global path planning algorithm for space manipulators which can plan a moving path on a space station with a minimum total energy demand. Different from existing approaches, we emphasize both the use of the proposed low energy demand gaits and the gaits composition during the path planning process. To evaluate the performance of the proposed gaits and path planning algorithm, numerous simulations are performed. Results show that the energy demand of both the proposed gaits and the resultant moving path is also minimum.
\end{abstract}

\section{Introduction}

The application of robots in space exploration becomes more advanced. For extravehicular activities (EVA) in low Earth orbit, most of them are designed as a chain-like manipulator which is suitable in performing tasks such as mechanical assistance, capturing operations, monitoring, and satellite maintenance [1]. Shuttle Remote Manipulator System (SRMS) [2, 3], Engineering Test Satellite No. 7 (ETS-VII) [4], the main arm (MA) and the small fine arm (SFA) of Module Remote Manipulator System (JEMRMS) [5], European Robotic Arm [6] and Space Station Remote Manipulator System (SSRMS), or Canadarm 2 [7] are typical examples for space manipulators. In addition to manipulation tasks, they are capable of navigating on the exterior of a space station. For the locomotion on a space station, two major gaits are commonly used which are inchworm gait and turnaround gait. Canadarm 2 is a typical example which uses inchworm gait for locomotion. Different from the inchworm gait, the turn around gait is another commonly used gait in conventional truss climbing robots such as Shady, Shady 3D, and Handbot [8-10].
For the conventional long limbs design of space manipulators, a larger joint torque is required to drive the arm's swing motion in conventional gaits. As a result, these conventional gaits are regarded as a high power demand motion. Since the energy source of a manipulator is from the attached space station, the minimization of energy consumption of a space manipulator is crucial to maintain normal functionalities of a space station. Without modifying the joint configuration of existing manipulators, we propose novel gaits for space manipulators by equipping a new gripping mechanism which combines the wheels locomotion in conventional grippers. For linear locomotion, it can eliminate the swing motions in conventional gaits. For turning motion, the range of swing motions is reduced by the wheels locomotion. Different from the Mobile Transporter of Mobile Servicing System (MSS), the proposed concept allows a manipulator to transit between trusses. In fact, several benefits can also be achieved. For example, the complexity of docking process during the exchange of gripping base can be simplified. As a result, the navigation efficiency can be enhanced. Also, the additional redundancy of the manipulator's mobility allows a fine positioning capability when it moves on a truss. 
Based on our previous research, we developed a gripping mechanism, Movable Gripper (MovGrip), which combines the concepts of active wheels in traditional parallel grippers [11]. With the use of MovGrip, a frame climbing robot, Frambot, is also developed which illustrates the methodology of using wheels in truss climbing [12]. With the advantages of MovGrip, one of the contributions in this paper is to propose novel gaits for space manipulators in minimizing the energy demand on a space station. To study the energy demand of different gaits, we extend the conventional modeling method to formulate the dynamic coupling effect during wheels locomotion. Based on the system's dynamic model, the proposed gaits are simulated and the corresponding energy demand is analyzed.

With the use of the proposed gaits, we further develop a global path planning algorithm for space manipulators which can plan a moving path on a space station with a minimum total energy demand. For conventional path planning algorithms, they are highlighted by many researchers in the category of planar mobile robots. Fernandez et al. proposed a path planning algorithm for mobile robot navigation with the use of a multicriteria path planar in 1999 [13]. In 2001, Gemeinder and Gerke proposed a genetic-algorithm(GA-) based robot path planning algorithm [14]. Wang et al. developed a path planning algorithm in 2008 which also considers the lifetime of a mobile robot [15]. In 2009, Ooi and Schindelhauer proposed a path planning algorithm to find a solution path for mobile robots with an optimal energy consumption for both robot's mobility and communication [16]. Liu and Sun proposed a path planning algorithm using $A^{*}$ algorithm with a minimum energy consumption in 2011 [17].

For the above algorithms, they mainly focus on the path planning problems whose workspace is limited on a $2 \mathrm{D}$ plane. Although the properties of a mobile robot and its working environment are studied, the robot's orientation after each move is not considered. For instance, space manipulators possess a 3D workspace. For a given current and target points pair, different manipulator's orientations on a truss can result in a different energy demand to reach the target point. Also, most of their works focused on the minimization of the path length as the energy consumption optimization. However, two paths with the same path length may have different energy demands. In fact, it depends on the motions composition in reaching a target position. Since the energy demand of each individual locomotion of a mobile robot can be different, the search of a solution path should also consider the composition of motion primitives.

Besides the planar mobile robots, the optimization problem is also addressed by researchers in climbing robots. For example, Balaguer proposed a path planning algorithm for ROMA which is a frame climbing robot developed to travel into complex metallic-based environments for inspection, assembly, and maintenance $[18,19]$. For different climbing aspects such as linear truss climbing, turning, and transition between surfaces, different climbing methodologies are considered and the one with a minimum energy demand is selected as the climbing motion for ROMA in that climbing aspect. Although the motion primitives are preselected with
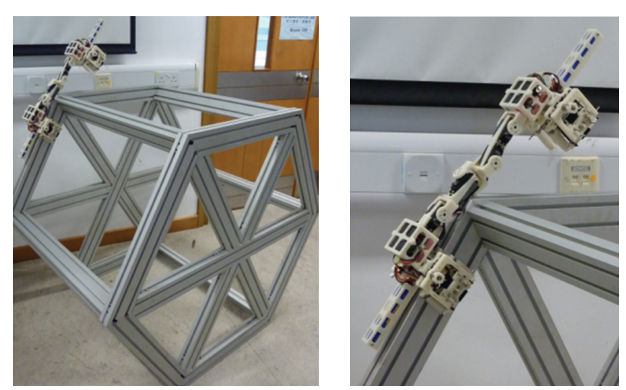

FIGURE 1: The prototype of Frambot.

a minimum energy demand, the composition of motion primitives in a climbing path is still not considered.

In this paper, the proposed path planning algorithm considers the composition of the proposed gaits in planning a moving path and the optimization is achieved by GA. To enhance the performance of the conventional GA, a novel genetic operator, Planar- $\mathrm{NN}$ is proposed and the design of Planar-NN, is based on the feature of the energy demands of the proposed gaits. The working principle of Planar-NN is to search a solution path with more motion primitives which contribute a lower energy demand. With this, the total energy demand of a solution path not only can be reduced by the use of motion primitives with a minimum individual energy demand, but also can further be minimized by focusing on the composition of motion primitives in the path.

The remaining of this paper is organized as follows. Section 2 gives a brief design description on our robot prototype, Frambot, including the robot's specifications, design of Movable Gripper (MovGrip), and locomotion. The conventional and proposed gaits for space robotic arms are illustrated in Section 3. Section 4 first describes methodology to model the dynamic coupling effect during wheels navigation. After that, the energy demand of the proposed gaits are simulated and analyzed. The global path planning algorithm is illustrated in Section 5. Finally, contributions of this paper are summarized in Section 6.

\section{Design of Frambot}

In this section, the design of a novel frame climbing robot (Frambot) is presented which aims to give a brief introduction on the design and use of a movable gripper in truss climbing. The working principle of the movable gripper on Frambot is first described. The major specifications of Frambot are also summarized. After that, the climbing motions of Frambot are illustrated. Details of the design and performance of Frambot are presented in $[11,12]$ and this is not repeated here.

2.1. Mechanical Design. Figure 1 shows the current prototype of Frambot which consists of (a) body linkage and (b) movable gripper (MovGrip). For the body linkage of Frambot, it is a four degree of freedom (DOF) serial robot linkage actuated by digital servo motors. Table 1 summaries the major specifications of Frambot. 
TABLE 1: The specifications of frambot.

\begin{tabular}{lc}
\hline Weight & $1.71 \mathrm{~kg}$ \\
Dimension $(L, W, H)$ & $(72.5,21,14.5) \mathrm{cm}$ \\
Gripping width & $(3.0-4.5) \mathrm{cm}$ \\
Maximum gripping force & $\sim 45 \mathrm{~N}$ \\
Steering angle of wheels & 5 degrees \\
Diameter of wheels & $2 \mathrm{~cm}$ \\
Vertical climbing speed & $2.31 \mathrm{~cm} / \mathrm{s}$ \\
Horizontal climbing speed & $4.05 \mathrm{~cm} / \mathrm{s}$ \\
Upside-down climbing speed & $6.2 \mathrm{~cm} / \mathrm{s}$ \\
\hline
\end{tabular}

The structure of MovGrip is composed of two major parts, (a) gripper jaw and (b) parallel gripping mechanism (Figure 2). Different from conventional design, each of the gripper jaws is equipped with two wheels actuated by mini geared motors through a spur gears transmission. For the parallel gripping mechanism, it is actuated by two linear servo motors and the gripping force is generated by the compression of springs. To simplify the design, the wheels' traction of MovGrip is contributed by the gripping force.

2.2. Locomotion on a Truss. For the design of conventional space manipulators, their end effector is mainly designed for grasping and different locomotion can only be performed by the swing motion of their body linkage. With the use of MovGrip, wheels locomotion can be utilized to minimize the arm's swing motion. For linear locomotion, it is performed by the rotation of wheels in MovGrip and the arm's swing motion in conventional turnaround and inchworm gaits are therefore eliminated. Figures 3 and 4 illustrate the turning and exterior transition of Frambot on a hexagonal aluminum truss, respectively. Frambot first lifts up the front gripper by using the body linkage. After that, it uses wheels locomotion to move forward until the front gripper can form a grasp on the target truss. Once a grasp is formed, the rear gripper is opened and lifted up. Similarly, Frambot uses wheels locomotion to climb forward until there is enough space for it to lower the rear gripper.

\section{Design of Locomotion}

One of the major contributions in this paper is to propose new gaits for space manipulators with an open chain configuration. For its gripping mechanism, it is assumed to be the concept presented in previous section. By this, the gripper not only provides a grasp on a truss, but also allows a linear locomotion along the truss by the embedded active wheels. Under this configuration, both conventional and proposed gaits can be realized and compared unbiasedly. For simplicity, we focus on the motion of a cubic truss structure which is symmetric about its principal axes. With this, the moment of inertia about the three axes is identical which simplifies the energy demand analysis.

3.1. Joint Configurations. Aforementioned, a space station is first regarded as a cubic truss. For the locomotion on a truss,

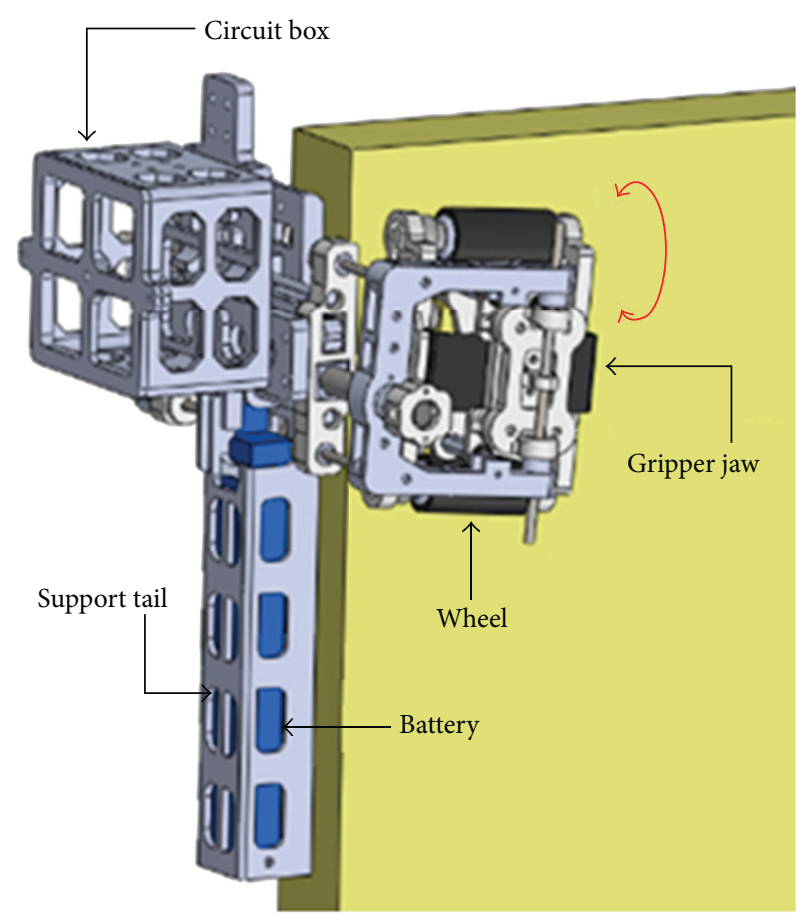

Figure 2: The design of MovGrip.

not all the joints of a seven-DOF space manipulator are involved. Therefore, the body of a space manipulator is defined as a five-DOF chain-like manipulator with its two ends equipped with MovGrip (Figure 5). It is a Roll-PitchPitch-Pitch-Roll configuration which can be considered as a simplified version of Canadarm 2 and ERA.

3.2. Linear Locomotion. For linear locomotion, the most commonly used gaits are turnaround and inchworm gaits [20]. Figure 6 illustrates the turnaround gait and the cross marked at the upper gripper indicates a closed grip. Firstly, the robot forms a grasp on a truss by the upper gripper and the other one is lifted up through the rotation of the three pitch joints (P2-P4). After that, the robot arm swings from back to front by using the roll joint (R5). Finally, the free gripper is lowered down and forms a grasp on the truss. The motion continues by repeating the above motion steps.

For inchworm gait, it is realized by using the three pitch joints (Figure 7). Firstly, the upper gripper forms a grasp on the truss and the lower one opens. After that, the three pitch joints (P2-P4) rotate so that the robot arm folds. Then, the lower gripper closes and the other one opens. Finally, the three pitch joints rotate so that the robot arm unfolds and the front gripper forms a grasp on the truss.

For the proposed locomotion, we propose the use of wheels locomotion to reduce the swing motions of a space manipulator. Since the manipulator does not move with respect to the gripper, the arm's swing motion is eliminated. Hence, the energy demand is converted to the rotation of wheels in the grippers. 


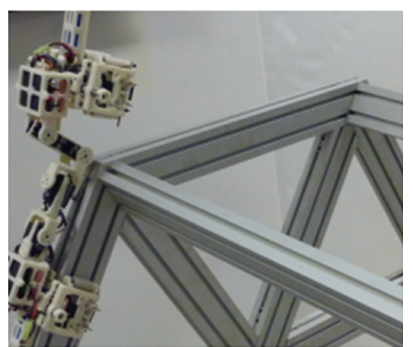

(a)

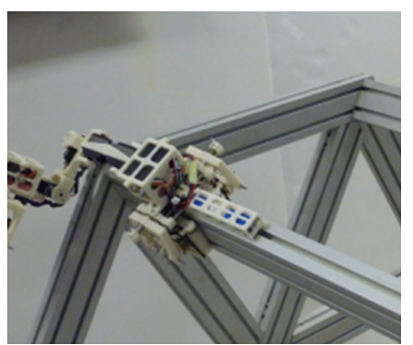

(e)

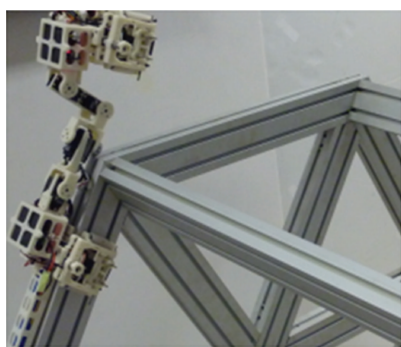

(b)

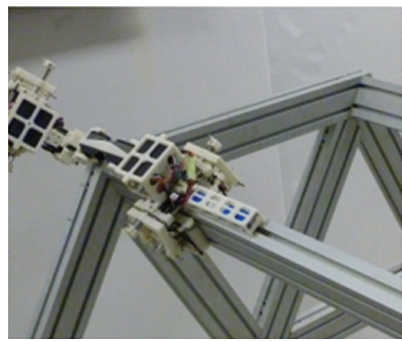

(f)

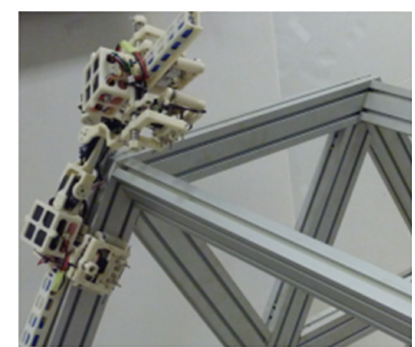

(c)

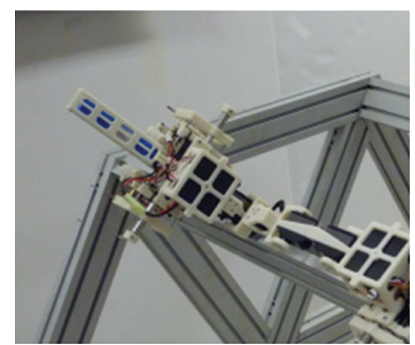

(g)

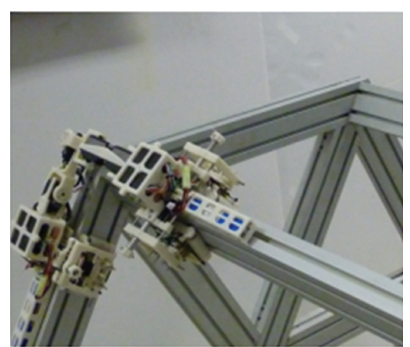

(d)

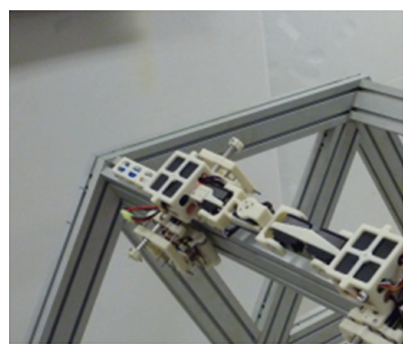

(h)

Figure 3: The turning motion of Frambot.

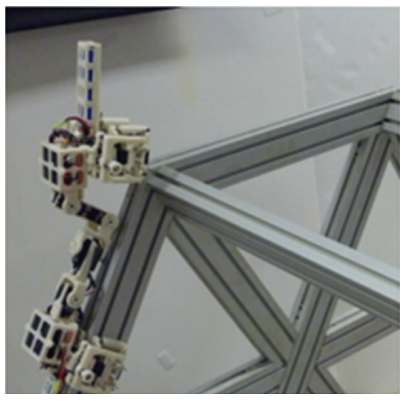

(a)

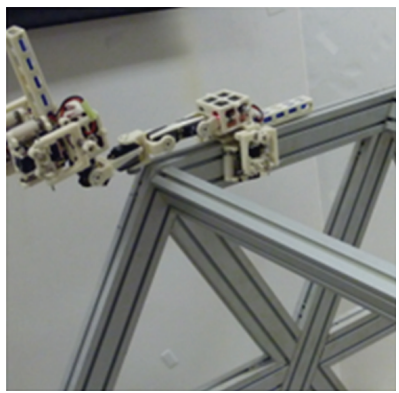

(d)

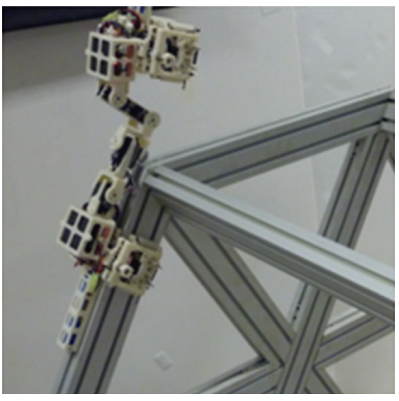

(b)

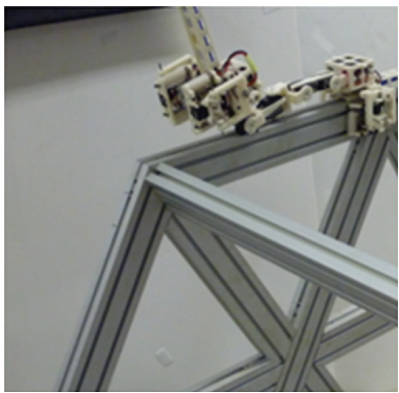

(e)

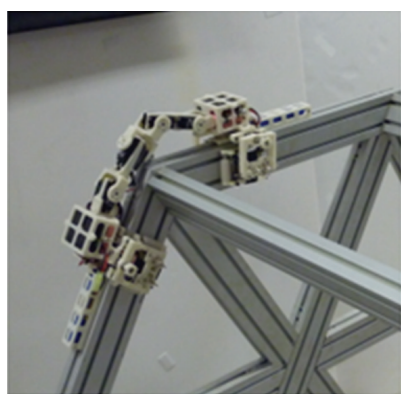

(c)

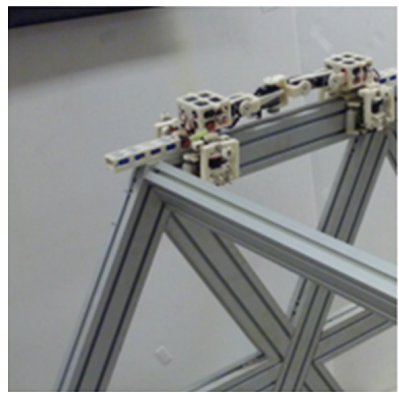

(f)

FIgURE 4: The exterior transition of Frambot.

3.3. Turning. Figure 8 illustrates the gait for turning. Firstly, the robot forms a grip by the front gripper and the other one is lifted up by the three pitch joints (P2-P4) (a). After that, the robot arm swings clockwise from the back to the left side by the roll joint (R5). The three pitch joints then rotate which lowers the free gripper (b). It grips the left truss and the other gripper opens. After that, the free gripper is lifted by the pitch joints and the robot arm swings until the free gripper is in a position above the left truss (c). Finally, the free gripper is lowered and a closed grasp is formed on the truss (d).

Figure 9 illustrates the proposed turning motion. Firstly, the robot forms a grasp by the rear gripper and the other one is lifted up by the three pitch joints (P2-P4) (a). After that, the robot moves forward by using wheels locomotion (b). The robot arm then swings to the left side by the roll joint (R1). The three pitch joints rotate which lowers the free gripper (c). It 


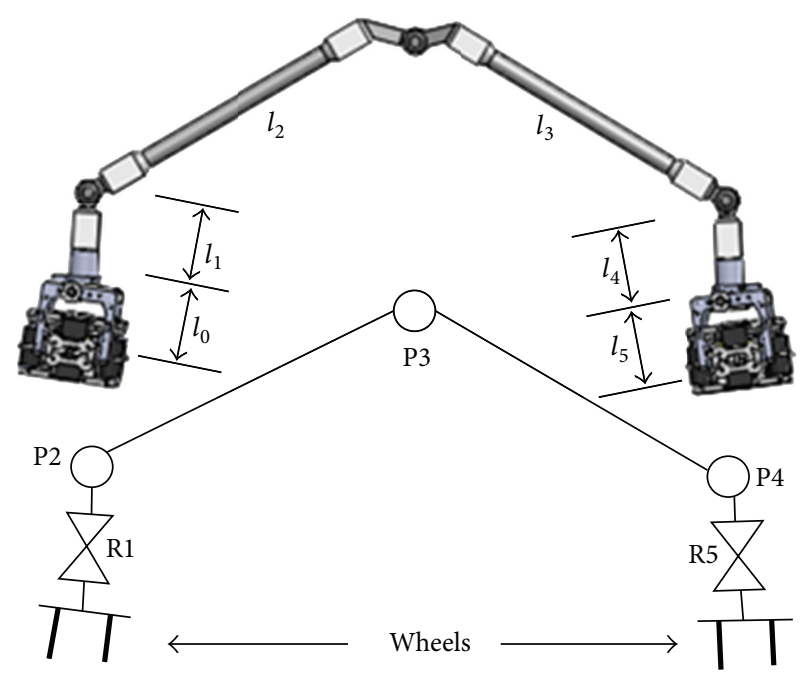

FIGURE 5: The joint configuration of the manipulator.

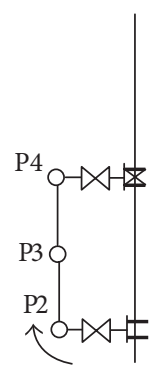

(a)

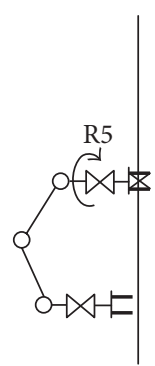

(b)

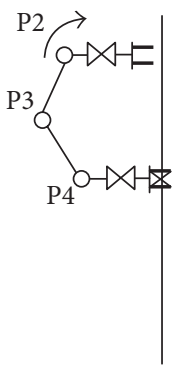

(c)

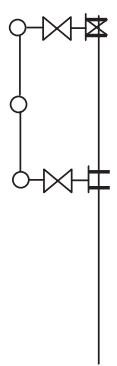

(d)
FIgURE 6: The gait for turn around gait.

grips the left truss and the other gripper then opens. After that, the free gripper is then lifted by the pitch joints and the robot arm swings until the free gripper is above the left truss (d). Finally, the robot moves forward by using wheels locomotion until there is enough space for it to lower the free gripper (e) and (f).

As an alternative of the turning gait, space manipulators can perform inchworm gait to replace the wheels locomotion in the proposed turning gait. Since the notion of inchworm gait is illustrated before, the use of inchworm gait in truss turning is not repeated here. When compared with the conventional turning gaits, there are two common motion steps which are the target posture of the motion steps (c) and (f). For the proposed turning gait, it uses wheels locomotion to reduce the rotation of roll joints in conventional gaits.

3.4. Exterior Transition. Figure 10 shows the conventional gait for exterior transition by using the turnaround gait. Firstly, the robot forms a grasp by the upper gripper and the other one is lifted up by the three pitch joints (P2-P4) (a). After that, the robot arm swings from back to the front by the roll joint (R5) (b). Then, the pitch joints rotate so that the free gripper can form a grasp on the exterior of the truss (c). The free gripper closes and the other gripper then opens.

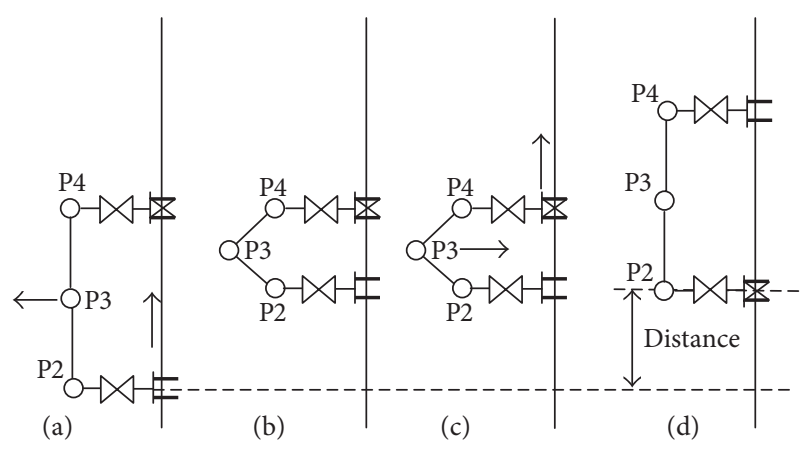

FIGURE 7: The gait for inchworm gait.

Similarly, the robot arm swings up by the pitch joints (d) which aims to make the whole robot above the exterior truss. After that, the roll joint (R1) rotates which swings the robot arm forward (e). Finally, the free gripper is lowered and a closed grasp is formed on the truss (f).

Figure 11 shows the proposed gait for exterior transition. Firstly, the robot forms a grasp by using the lower gripper and the other one is lifted up by the three pitch joints (P2P4) (a). After that, the robot moves forward by using wheels locomotion (b). Then, the pitch joints rotate so that the free gripper can grasp the exterior of the truss (c). The free gripper closes and the other gripper then opens. Similarly, the robot arm swings up by the pitch joints (d) which aims to make the whole robot above the exterior truss. Finally, the robot moves forward by using wheels locomotion until there is enough space for it to lower the free gripper (e) and (f).

Similar to the turning gait, space manipulators can also perform inchworm gait to replace the wheels locomotion in the proposed transition gait and it is not illustrated here. Also, there are two common motion steps for the conventional and proposed transition gaits which are the target posture of the motion steps (Figures 11(c) and 11(f)). For the proposed gait, it minimizes the rotation of roll joints by driving the robot forward using wheels locomotion.

\section{Analysis of Energy Demand}

For the energy demand of a gait, it is defined as the energy required to perform that gait. For the joint's motion, the energy demand, " $E_{i}$ " of the $i$ th joint can be formulated as the time integration of the joint torque, " $\tau_{i}(t)$," and angular velocity, " $\dot{q}_{i}(t)$, , multiplication as

$$
E_{i}=\int \tau_{i}(t) \cdot \dot{q}_{i}(t) d t
$$

Similarly, the energy demand, " $\left(E_{i}^{\prime}\right)$," for the wheels locomotion is the time integration of the wheels traction force, " $F_{i}(t)$," and linear velocity, " $V_{G}(t)$," multiplication as

$$
E_{i}^{\prime}=\int F_{i}(t) \cdot V_{G}(t) d t .
$$

The total energy demand of a gait can be computed as the sum of all individual energy demands. To compare the 


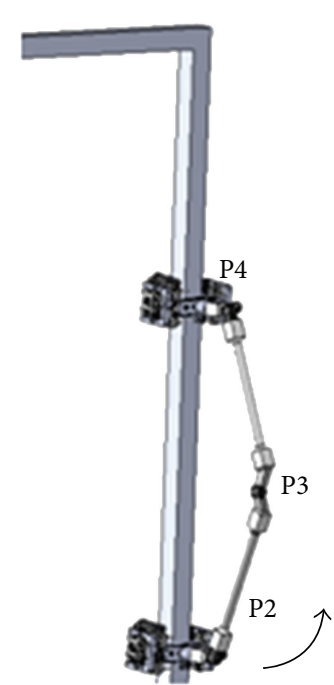

(a)

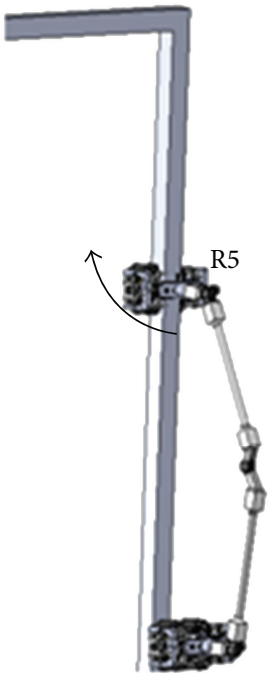

(b)
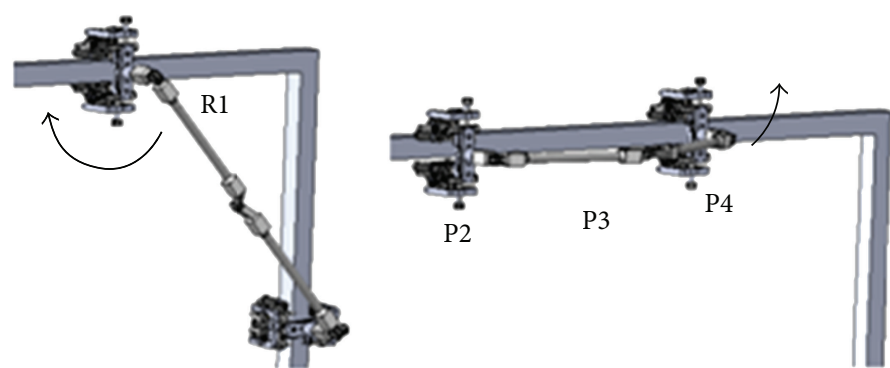

(c)

(d)

Figure 8: The conventional gait for turning.

energy demand of different gaits of a space manipulator, the kinematics, momentums, and dynamics of a space robotic system provide the fundamental approach. Because of this, this section first presents the modeling of a space robotic system. After that, the energy demand of different gaits is analyzed.

4.1. Modeling of a Space Robotic System. Since the formulation of the dynamic coupling effect of a space manipulator with one end fixed on a space station is commonly established [21-23], this paper only focuses on the approach to model the dynamic coupling effects under wheels locomotion. A general model for a space robotic system is first described. After that, the system's kinematics and momentum relationships are presented. To determine the system's dynamic equations, Lagrangian formulation is utilized.

Figure 12 shows a general model of a space robotic system. The space manipulator consists of $n$ joints which are numbered from 1 to $n$. Since the manipulator is mounted on a free floating space station, the whole system is regarded as a free flying chain-like system with $n+1$ links. As the space station can translate and rotate freely about the inertial frame axes, the total DOF of the system is $n+6$.

For the system modeling, three coordinate frames are first introduced which include (a) inertial frame, (b) manipulator frame, and (c) base frame. For the inertial frame, its origin is fixed in the space. For the manipulator frame and base frame, their origins are located at the mass center of the manipulator and base, respectively. During the modeling, a number of notations are defined as follows:

$$
\begin{aligned}
& q \in R^{n}: \text { joint variable vector }\left(=\left[q_{1}, q_{1}, \ldots, q_{n}\right]^{T}\right), \\
& \dot{q} \in R^{n}: \text { joint angular velocity vector, } \\
& \tau \in R^{n}: \text { joint torque vector }\left(=\left[\tau_{1}, \tau_{1}, \ldots, \tau_{n}\right]^{T}\right), \\
& R_{b} \in R^{3}: \text { position vector of the base, } \\
& R_{i} \in R^{3}: \text { position vector of the mass center of link } i, \\
& R_{G} \in R^{3}: \text { position vector of the manipulator's mass } \\
& \text { center center, }
\end{aligned}
$$

$R_{i / b} \in R^{3}$ : position vector of the link $i$ w.r.t. to the base frame,

$R_{G / b} \in R^{3}$ : position vector of the manipulator's mass center $i$ with respect to the base frame,

$V_{b} \in R^{3}$ : linear velocity of the base,

$\Omega_{b} \in R^{3}$ : angular velocity of the base,

$m_{b}$ : mass of the base, 


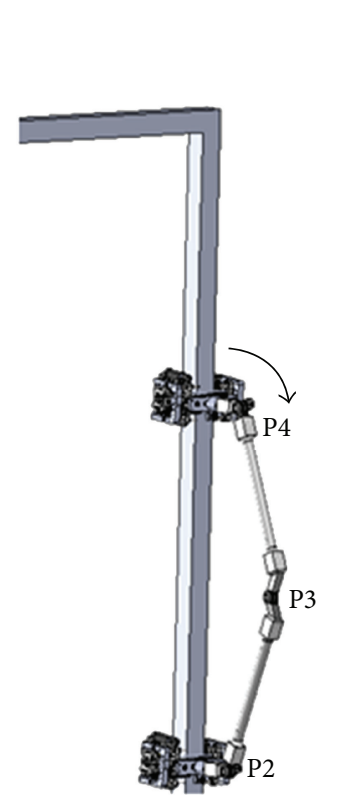

(a)

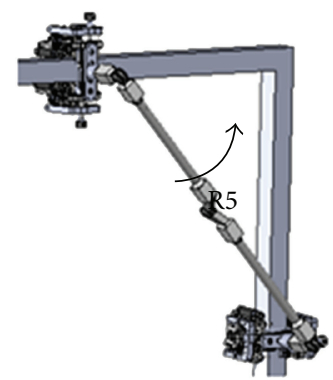

(d)

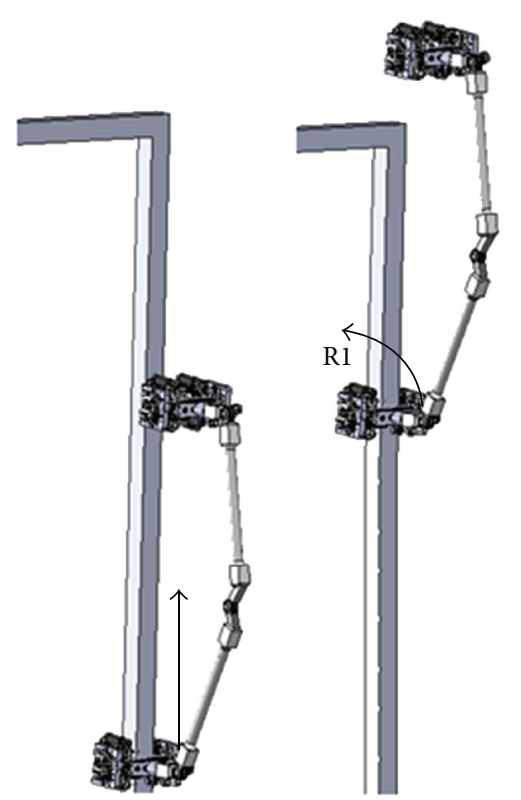

(b)

(c)

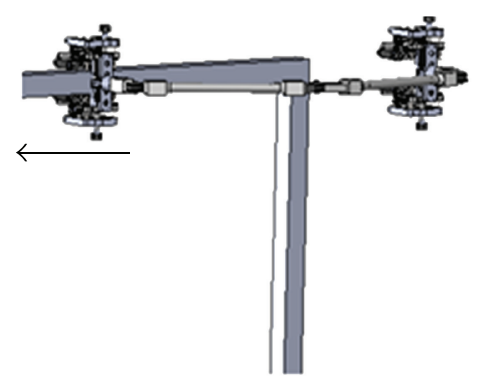

(e)

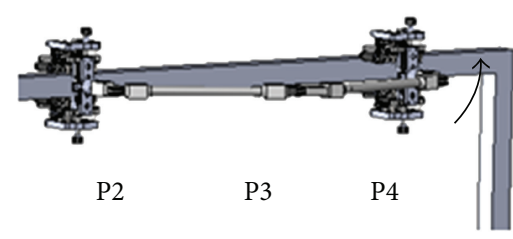

(f)

FIGURE 9: The proposed turning motion.

$m_{T}$ : total mass of the system,

$m_{G}$ : total mass of the manipulator,

$I_{G} \in R^{3 \times 3}$ : inertial tensor matrix of the manipulator with respect to its mass center,

$I_{b} \in R^{3 \times 3}$ : inertial tensor matrix of the base with respect to its mass center,

$U \in R^{3 \times 3}: 3 \times 3$ identity matrix.

All vectors are described with respect to the inertial coordinate system unless another coordinate system is mentioned and $i=0,1, \ldots, n$. Here, two matrix functions are defined for a vector $r=\left[r_{x}, r_{y}, r_{z}\right]^{T}$ as follows:

$$
\begin{aligned}
{[r \times] } & \equiv\left[\begin{array}{ccc}
0 & -r_{z} & r_{y} \\
r_{z} & 0 & -r_{x} \\
-r_{y} & r_{x} & 0
\end{array}\right], \\
{[G(r)] } & \equiv[r \times]^{T}[r \times] \\
& =\left[\begin{array}{ccc}
r_{y}^{2}+r_{z}^{2} & -r_{x} r_{y} & -r_{x} r_{z} \\
-r_{x} r_{y} & r_{x}^{2}+r_{z}^{2} & -r_{y} r_{z} \\
-r_{x} r_{z} & -r_{y} r_{z} & r_{x}^{2}+r_{y}^{2}
\end{array}\right] .
\end{aligned}
$$

In addition, several assumptions are made. First of all, all links are rigid bodies and there are no external forces 


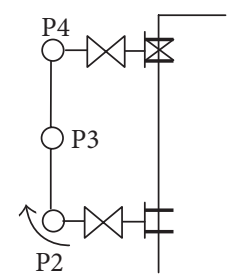

(a)

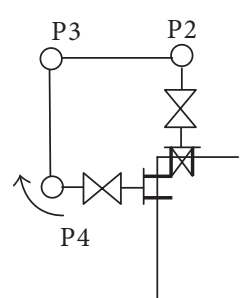

(d)

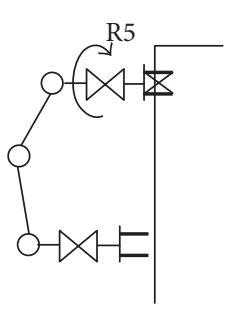

(b)

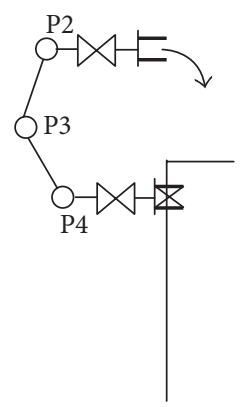

(c)

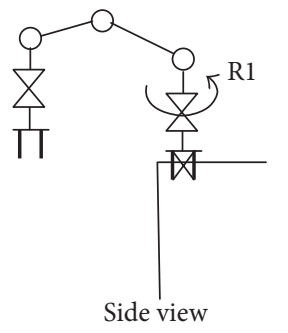

(e)

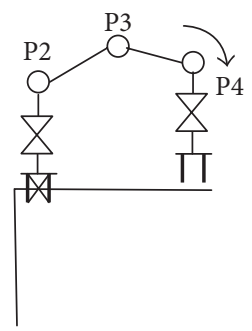

(f)
FIGURE 10: The conventional gait for exterior transition.

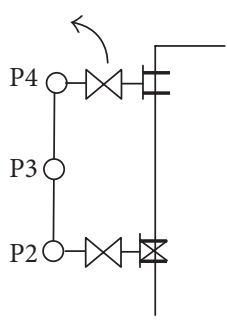

(a)

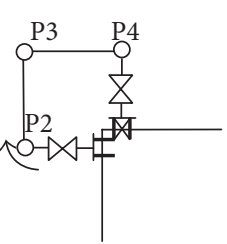

(d)

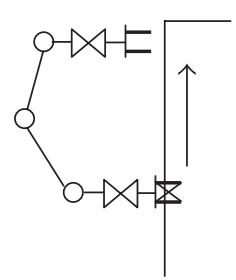

(b)

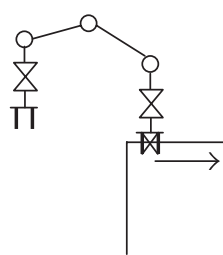

(e)

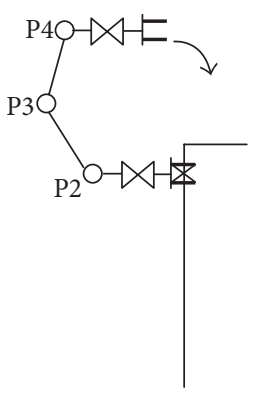

(c)

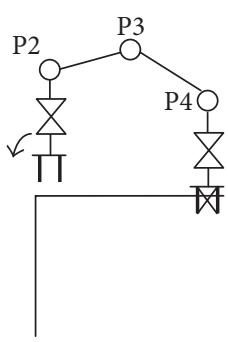

(f)
FIGURE 11: The proposed gait for exterior transition.

and torques acting on the system. As a result, the total potential energy of the system is equal to zero. During wheels locomotion, it is assumed that the configuration of the manipulator is maintained (i.e., $\dot{q}=\ddot{q}=0$ ). Also, the modeling is with respect to the inertial frame unless other coordinate frame is specified.

4.2. Kinematics. To model the kinematics of the system, the manipulator is first visualized as a prismatic joint and the

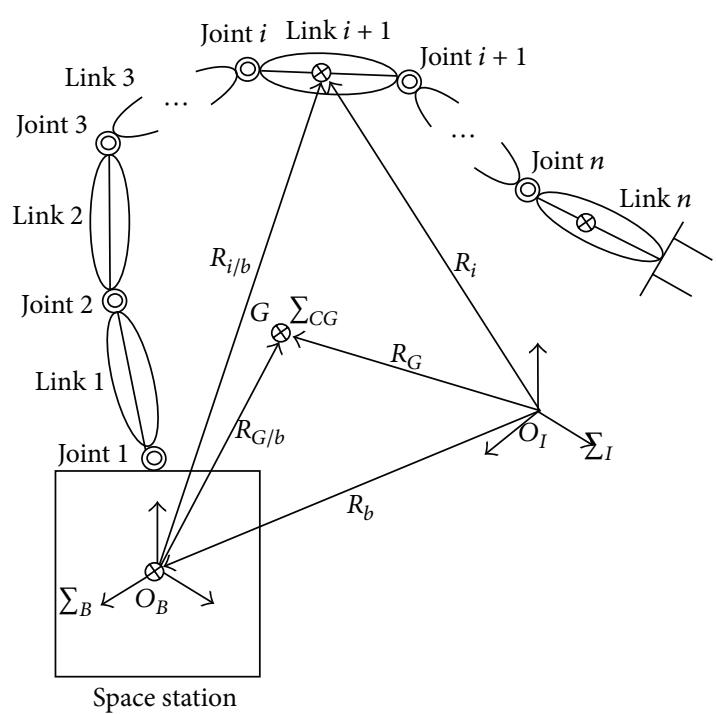

FIgURE 12: A general model of a space robotic system.

closed MovGrip is the gripping point on the base. During wheels locomotion, the resultant wheels traction becomes the pushing force of the prismatic joint acting on the base and the gripping point is moving together with wheels locomotion. Since the manipulator does not move with respect to the gripper, the position vector of the manipulator's mass center can be formulated as follows:

$$
R_{G}=R_{b}+R_{G / b} .
$$

By taking time derivative on both sides, we can derive the system's kinematics relationship as follows:

$$
V_{G}=V_{b}+\Omega_{b} \times R_{G / b}+v_{G},
$$

where $v_{G}$ is the linear velocity of the manipulator under wheels locomotion. $V_{G}$ is the linear velocity of the manipulator's mass center with respect to the inertial frame. Since the whole system rotates together under wheels locomotion, both the manipulator and base have the same angular velocity (i.e., $\left.\Omega_{G}=\Omega_{b}\right)$.

4.3. Linear and Angular Momentums. Aforementioned, it is assumed that there are no external forces and torques acting on the system, and the linear and angular momentums of the system are conserved as

$$
\begin{gathered}
P=m_{b} V_{b}+m_{G} V_{G}, \\
L={ }^{B} I_{b} \Omega_{b}+m_{b} R_{b} \times V_{b}+{ }^{B} I_{G} \Omega_{b}+m_{G} R_{G} \times V_{G} .
\end{gathered}
$$

From the kinematics relationship, we express the variables $R_{G}$ and $V_{G}$ in terms of $V_{b}, \Omega_{b}$ and $v_{G}$. By rearranging the variables of the momentum equations, they can derive the following momentum relationship:

$$
\left[\begin{array}{l}
P \\
L
\end{array}\right]=\left[\begin{array}{cc}
M_{V b} & M_{V_{b} \Omega_{b}} \\
M_{V_{b} \Omega_{b}}^{T} & M_{\Omega_{b}}
\end{array}\right]\left[\begin{array}{c}
V_{b} \\
\Omega_{b}
\end{array}\right]+\left[\begin{array}{l}
M_{V_{b} v_{G}} \\
M_{\Omega_{b} v_{G}}
\end{array}\right] v_{G},
$$


where

(i) $M_{V_{b}} \equiv \sum_{i=0}^{n} m_{i} U=m_{T} U \in R^{3 \times 3}$

(ii) $M_{V_{b} \Omega_{b}} \equiv m_{G}\left[R_{G / b} \times\right] \in R^{3 \times 3}$

(iii) $M_{\Omega_{b}} \equiv\left\{I_{G}+m_{G}\left[G\left(R_{G / b}\right)\right]\right\}+I_{b} \in R^{3 \times 3}$

(iv) $M_{V_{b} v_{G}} \equiv m_{G} J_{m T_{G}} \in R^{3 \times 1}$

(v) $M_{\Omega_{b} v_{G}} \equiv m_{G}\left[R_{G / b} \times\right] J_{m T_{G}} \in R^{3 \times 1}$.

$J_{m T_{G}}$ is the linear Jacobian matrix of the manipulator under wheels locomotion and is defined as follows:

$$
J_{m T_{G}}={ }^{I} \operatorname{Rot}_{G} z_{G} \text {. }
$$

${ }^{I} \operatorname{Rot}_{G}$ is the rotation matrix from the manipulator frame to the inertial frame and $z_{G}$ is the $z$-axis of the manipulator frame which indicates the direction of gripper's movement. If the system is initially at rest, the following constraints can be obtained:

$$
\left[\begin{array}{cc}
M_{V b} & M_{V_{b} \Omega_{b}} \\
M_{V_{b} \Omega_{b}}^{T} & M_{\Omega_{b}}
\end{array}\right]\left[\begin{array}{c}
V_{b} \\
\Omega_{b}
\end{array}\right]+\left[\begin{array}{l}
M_{V_{b} v_{G}} \\
M_{\Omega_{b} v_{G}}
\end{array}\right] v_{G}=\left[\begin{array}{l}
0 \\
0
\end{array}\right] .
$$

4.4. Dynamics. For the system's dynamic modeling, the conventional Lagrangian approach is utilized. Since the total potential energy of the system is zero, the total energy of the system during wheels locomotion is the sum of translational and rotational kinetic energy $(K)$ of all bodies in the system as

$$
K \equiv \frac{1}{2}\left(m_{b} V_{b}^{T} V_{b}+\Omega_{b}^{T} I_{b} \Omega_{b}+m_{G} V_{G}^{T} V_{G}+\Omega_{b}^{T} I_{G} \Omega_{b}\right) .
$$

Similar to the momentum equations, the energy equations can be expressed in terms of $V_{b}, \Omega_{b}$, and $v_{G}$ as

$$
\begin{gathered}
K=\frac{1}{2} \dot{\theta}^{T} M(\theta) \dot{\theta} \\
M(\theta)=\left[\begin{array}{ccc}
M_{V_{b}} & M_{V_{b} \Omega_{b}} & M_{V_{b} v_{G}} \\
M_{V_{b} \Omega_{b}}^{T} & M_{\Omega_{b}} & M_{\Omega_{b} v_{G}} \\
M_{V_{b} v_{G}}^{T} & M_{\Omega_{b} v_{G}}^{T} & M_{v_{G}}
\end{array}\right] \in R^{7 \times 7}, \\
\dot{\theta}^{T}=\left[\begin{array}{lll}
V_{b} & \Omega_{b} & v_{G}
\end{array}\right] \\
M_{v_{G}}=m_{G} J_{m T_{G}}^{T} J_{m T_{G}} \in R^{1 \times 1} .
\end{gathered}
$$

The matrix $M(\theta)$ is the system inertial matrix which is positive definite and symmetric. By using the Lagrangian formulation

$$
\frac{d}{d t}\left(\frac{\partial K}{\partial \dot{\theta}}\right)-\frac{\partial K}{\partial \theta}=\tau,
$$

the dynamic model of the system can be derived as

$$
M(\theta) \ddot{\theta}+C(\theta, \dot{\theta}) \dot{\theta}=\tau
$$

where $\tau$ is the generalized force vector and $C(\theta, \dot{\theta})$ is the nonlinear centrifugal and Coriolis forces as

$$
C(\theta, \dot{\theta}) \dot{\theta}=\dot{M}(\theta)-\frac{\partial}{\partial \theta}\left(\frac{1}{2} \dot{\theta}^{T} M(\theta) \dot{\theta}\right) .
$$

TABLE 2: The system parameters for simulations.

\begin{tabular}{lccc}
\hline$l_{0}$ & $0.5 \mathrm{~m}$ & $I_{b x x}, I_{b y y}, I_{b z z}$ & $1.3599 \mathrm{~kg} \mathrm{~m}^{2}$ \\
$l_{1}$ & $0.5 \mathrm{~m}$ & $\left(I_{1 x x}, I_{1 y y}, I_{1 z z}\right)$ & $(1.3,1.3,0.1) \cdot 10^{-4} \mathrm{~kg} \mathrm{~m}^{2}$ \\
$l_{2}$ & $2 \mathrm{~m}$ & $\left(I_{2 x x}, I_{2 y y}, I_{2 z z}\right)$ & $(0.4,80.2,80.2) \cdot 10^{-4} \mathrm{~kg} \mathrm{~m}^{2}$ \\
$l_{3}$ & $2 \mathrm{~m}$ & $\left(I_{3 x x}, I_{3 y y}, I_{3 z z}\right)$ & $(0.4,80.2,80.2) \cdot 10^{-4} \mathrm{~kg} \mathrm{~m}^{2}$ \\
$l_{4}$ & $0.5 \mathrm{~m}$ & $\left(I_{4 x x}, I_{4 y y}, I_{4 z z}\right)$ & $(1.3,0.1,1.3) \cdot 10^{-4} \mathrm{~kg} \mathrm{~m}^{2}$ \\
$l_{5}$ & $0.5 \mathrm{~m}$ & $\left(I_{5 x x}, I_{5 y y}, I_{5 z z}\right)$ & $(1.3,1.3,0.1) \cdot 10^{-4} \mathrm{~kg} \mathrm{~m}^{2}$ \\
$m_{b}$ & $483.7 \mathrm{~kg}$ & $m_{1}$ & $15 \mathrm{~kg}$ \\
$m_{2}$ & $10 \mathrm{~kg}$ & $m_{3}$ & $10 \mathrm{~kg}$ \\
$m_{4}$ & $10 \mathrm{~kg}$ & $m_{5}$ & $15 \mathrm{~kg}$ \\
\hline
\end{tabular}

4.5. Simulation Study. For the analysis of energy demand of the proposed gaits, it is assumed that there are no external forces and torques acting on the system. For the space station, it is assumed to be a cubic truss structure which gives a symmetric property for simulations. Length of a side of the truss is 10 meters and the presented system dynamic model is utilized to compute the energy demand.

Both the proposed and conventional gaits are simulated and three different aspects are studied including (a) linear locomotion, (b) turning, and (c) exterior transition. For each of the three aspects, three gaits are considered including (a) turnaround gait, (b) inchworm gait, and (c) wheels locomotion. For fair comparison, the moving distance of different gaits in each of the three aspects is equal. Also, the time to perform different gaits in each of the three cases is fixed. Table 2 lists the system parameters for simulations.

Figure 13 shows the definition of the base, base frame, and robot initial and final configurations. For linear locomotion, the robot (marked with dotted line) is initially set at the corner of the edge A. It moves forward by using different gaits and stops at a distance of 2 meters away from the other end (corner G).

For the turning gaits at a truss corner, only the turn left gait is discussed and it is performed from edge A to edge B of the space station in Figure 13. The robot is initially set at a position where its front gripper $(f)$ is at a distance of 2.828 meters on edge A away from corner $G$. It performs the turning motion by using different gaits and stops at a position on edge $\mathrm{B}$ where its rear gripper $(r)$ is at a distance of 6.828 meters away from that corner. For the exterior transition at a truss corner, the motions are performed from edge A to edge $C$ of the space station in Figure 13. The robot is initial set at a position where its front gripper $(f)$ is at a distance of 2 meters on edge A away from corner $\mathrm{G}$. It performs exterior transition and stops at a position on edge $\mathrm{C}$ where its rear gripper $(r)$ is at a distance of 6 meters away from that corner.

Figures 14 and 15 show the angular displacement and torque profile of the inchworm gait, respectively. Since the distance travel of an inchworm gait is two meters, the two repeated patterns in Figure 14 indicate the two repetitions of the arm folding and unfolding motions. Also, only the three pitch joints (joints 2-4) are involved to perform the inchworm gait. Therefore, the torque profile of three pitch joints is nonzero. 


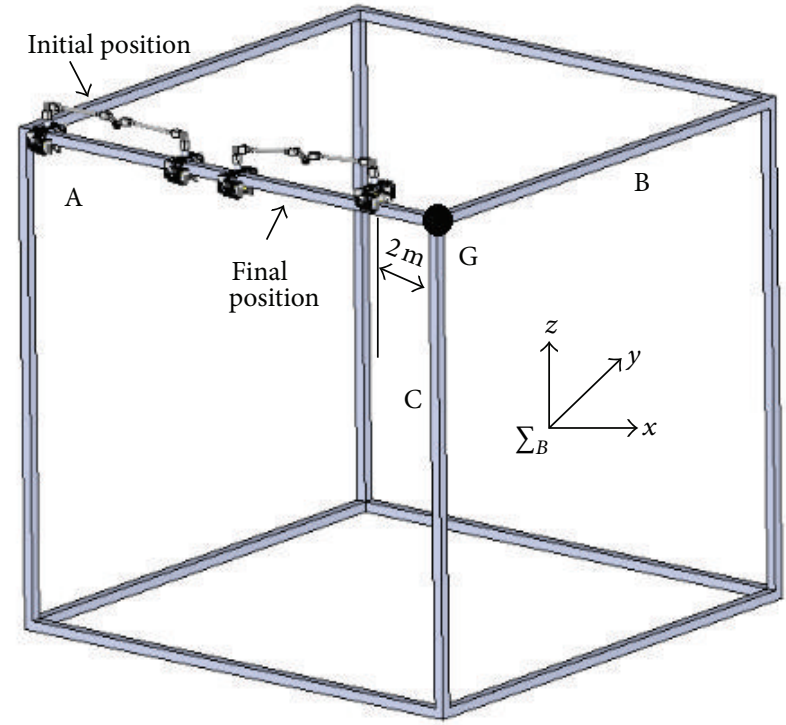

FIgURE 13: The space station, base frame, and robot initial and final configuration for wheels locomotion.

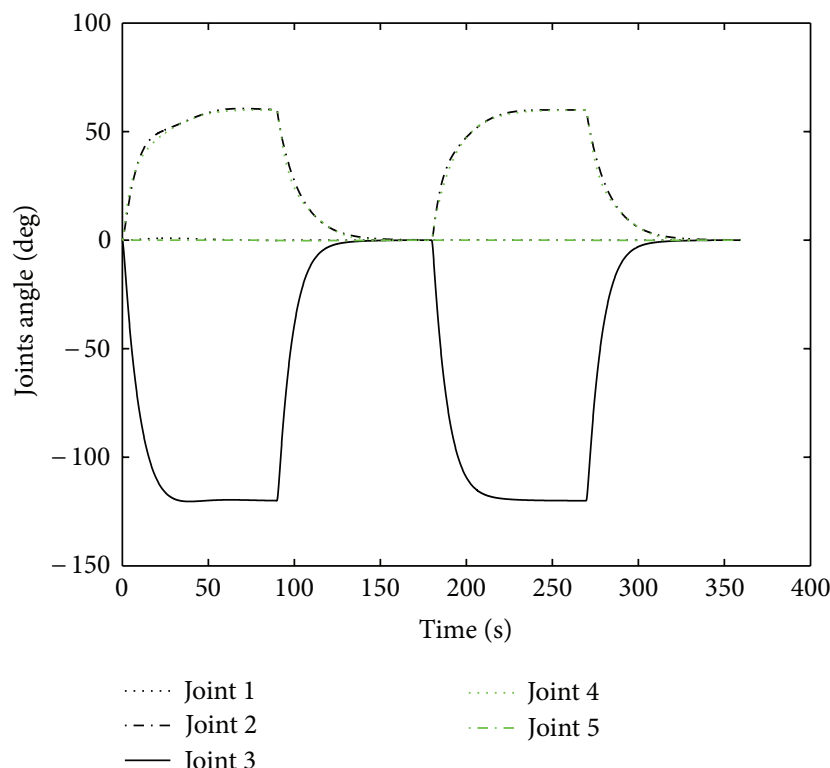

FIGURE 14: The joints' displacement profiles for the inchworm gait.

By using (1), the total energy demand of the inchworm gait is 2.0458 joules per meter $(\mathrm{J} / \mathrm{m})$. By using the similar approach, the total energy demand for the other turning and transition motions can be computed which are summarized in Table 3.

From the nature of the gaits, the arm folding and unfolding motions of the inchworm gait on the one hand require the largest joints movement when compared with the others. On the other hand, the distance travel in one motion step is the smallest. Given a certain operation time to complete the gait, the joints' torque and angular velocity are much larger

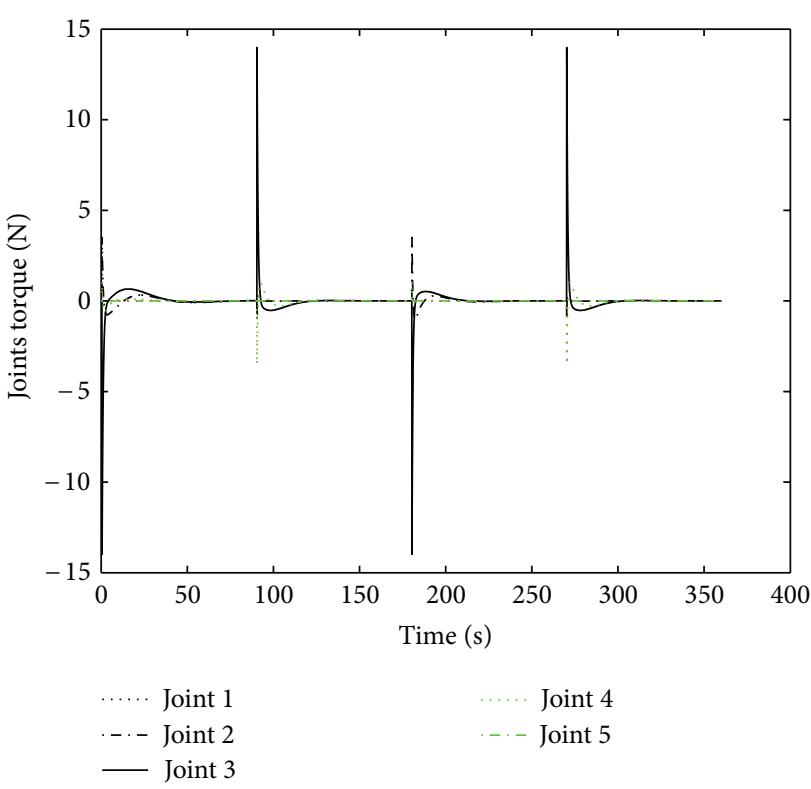

FIGURE 15: The joints' torque profiles for the inchworm gait.

TABLE 3: The total energy demand of different gaits.

\begin{tabular}{lccc}
\hline & Inchworm & Turn around & $\begin{array}{c}\text { Wheels } \\
\text { locomotion }\end{array}$ \\
\hline Linear & $2.0458 \mathrm{~J} / \mathrm{m}$ & $1.0097 \mathrm{~J} / \mathrm{m}$ & $0.0424 \mathrm{~J} / \mathrm{m}$ \\
Turning & $41.2473 \mathrm{~J}$ & $4.6744 \mathrm{~J}$ & $3.1689 \mathrm{~J}$ \\
$\begin{array}{l}\text { Exterior } \\
\text { transition }\end{array}$ & $17.3267 \mathrm{~J}$ & $9.3039 \mathrm{~J}$ & $3.7817 \mathrm{~J}$ \\
\hline
\end{tabular}

than the others. Under a constraint on the operation time, the inchworm gait is regarded as a high power demand motion.

For the wheels locomotion, the wheels' traction force is in the same direction as the movement. Also, all the mass is moving along a straight line during wheels locomotion. With a long limb design of conventional space manipulator, the use of wheels locomotion can avoid the arm's swing motion whose joints torque is high. Therefore, the use of wheels locomotion to reduce the joints movement allows an energy efficiency locomotion on a space station.

\section{Global Path Planning Algorithm}

For the proposed minimum energy demand-based path planning algorithm, the optimization goal is first defined. Given the robot's initial position and orientation, a list of check points (including the target point) and their position with respect to an inertial frame, the objective is to search a path which passes all the check points once and reaches the target point with a minimum energy demand. This can be visualized as a space manipulator is commanded to follow the solution path and visit a list of points for monitoring or maintenance tasks on a space station. Finally, it is commanded to reach a target point for charging or standby.

Figure 16 shows the geometry of a space station. The edges in the structure are the frame for grasping and the corners are 


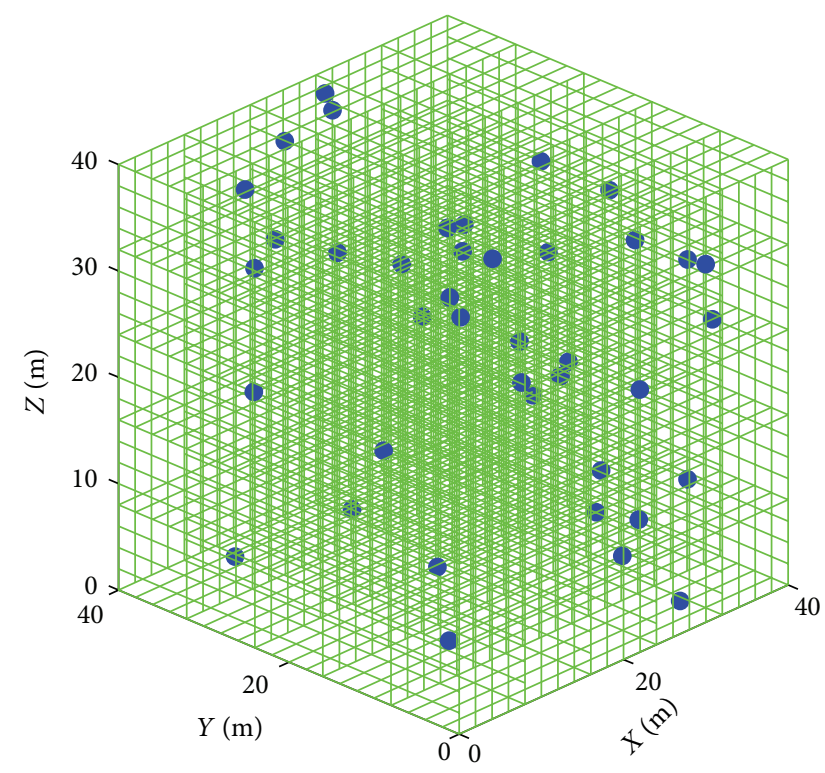

FIGURE 16: The geometry of a space station.

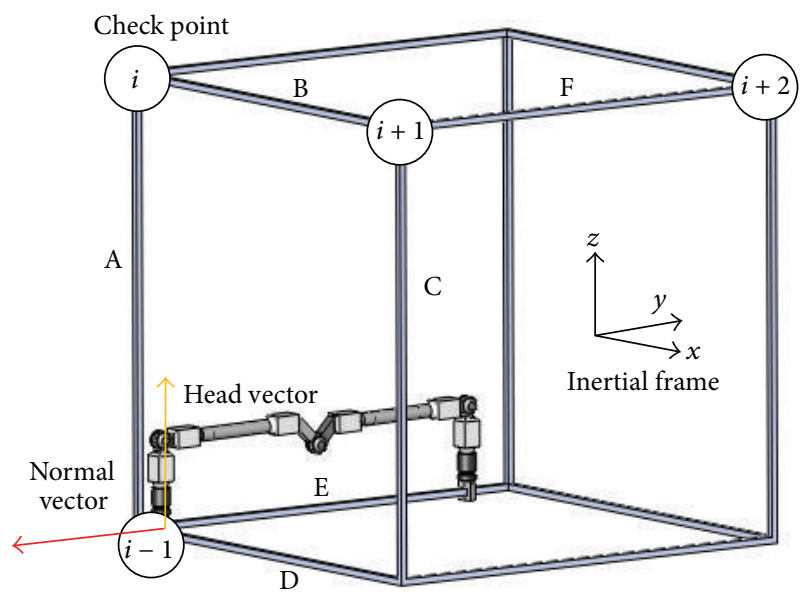

FIgURE 17: An example illustrating the computation of individual demand.

the possible check points. To travel all the check points along the solution path, a space manipulator is assumed to perform the basic motion primitives.

5.1. Representation of a Chromosome. In conventional GA, a chromosome is the basic element for genetic evolution. For the proposed algorithm, a chromosome represents the path for navigation. It is composed of $n$ genes and $n$ is equal to the total number of check points. Each of the genes is labeled with a unique integer which indicates the visiting order. Therefore, the integers in a chromosome describe the order of check points being visited.

5.2. Evaluation Function. In GA, chromosomes with a higher fitness (lower energy demand) with respect to the optimization goal are selected for genetic evolution. To measure the fitness of a chromosome, an evaluation function should be defined. In this algorithm, the optimization goal is to search an optimal path with a minimum energy demand. The fitness function is therefore defined as the total energy demand of the path. Since a chromosome is composed of genes representing the order of check points being visited, the total energy demand of a path is the summation of the individual energy demand of all consecutive gene pairs along the chromosome. Figure 17 shows a manipulator climbing on a truss structure as an example. It is currently at the check point $i-1$ and gripping on the edge A. To compute the energy demand for reaching the next check point, the current manipulator's orientation is considered. To represent the manipulator's orientation, two vectors are defined. They are the head and normal vectors which are labeled with orange and red colors, respectively. For the head vector, it indicates the current gripper direction after traveling from the point $i-2$ to $i-1$. If $i-2$ is the first point, it represents the initial orientation of the robot gripper. For the normal vector, it is perpendicular to the truss surface and pointing away from the robot gripper.

For the energy demand of a pair of check points, there are three major possible cases. The first one is the case when the line connecting them is parallel to one of the inertial frame axes. The points pair $i-1$ and $i$ in Figure 17 shows an example with the line jointing them is parallel to the $z$-axis. For the second case, two check points lie on a plane which is parallel to the plane formed by any two of the inertial frame axes. The points pair $i-1$ and $i+1$ shows an example with the plane parallel to the $x-z$ plane of the inertial frame. For the last case, the coordinate values of the two check points are different. The points pair $i-1$ and $i+2$ shows an example that does not satisfy the first two cases. For simplicity, this paper only illustrates the computation of individual energy demand of a points pair in the second case. Because a similar technique can be used for the other cases, it is not repeated here.

Assumed that the manipulator is currently at the check point $i-1$ and gripping on the edge $\mathrm{E}$, in this case, if its head and normal vectors are initially pointing to the negative $y$-axis and positive $z$-axis, respectively, there are two possible paths to travel from point $i-1$ to $i+1$. The first motion sequence contains interior transition, linear frame climbing, turn left, and linear frame climbing. The other motion sequence contains turn left, linear frame climbing, interior transition, and linear frame climbing. Since the energy demand of both paths is the same, the motion sequencing to travel from point $i-1$ to $i+1$ is chosen randomly in order to maintain the global search of a solution path in GA. The corresponding energy demand is computed as follows:

$$
E_{i-1}=E_{\text {interior }}+E_{\text {left }}+(\Delta z+\Delta x) \times E_{\text {linear }}
$$

$\Delta x$ and $\Delta z$ are the edge length between the two points along the $x$ and $z$ axes of the inertial frame, respectively. $E_{\text {linear }}, E_{\text {left }}$, and $E_{\text {interior, }}$, are the energy demand for the manipulator to move one meter forward, turn left, and perform interior transition, respectively. After computing the move to the next check point, the updated head and normal 
vectors of the manipulator become the current head and normal vectors. Although there are other possible cases for the initial condition of the manipulator in all the above three cases, similar techniques can be utilized and they are not repeated here for simplicity. Finally, the total energy demand to travel along a solution path is the sum of all individual energy demands and it is formulated as follows:

$$
E_{\text {Total }}=\sum_{i=1}^{n-1} E_{i} .
$$

$E_{i}$ is the energy demand for a manipulator to navigate from the $i$ th check point back to the $i+1$ th point.

5.3. Genetic Operators. From the energy demands of the motion primitives in Table 3, the total energy demand of a path is lower if it is composed of more planar (or linear) path segments. Because of this, the proposed path planning algorithm focuses on the searching of an optimal path which consists of more planar path segments. With the use of GA for optimization, we enhance the evolution process by developing a novel exchange mechanism for the genetic information of a chromosome. It is a genetic operator which promotes the path with planar (or linear) genes segments. It is called Planar-NN and NN means nearest neighbor.

Figure 18 illustrates the working principle of Planar-NN. A sample frame structure is shown and the orange dot indicates the initial position of the manipulator. The grey and blue dots are the intermediate and final check points, respectively. An elitist chromosome for genetic evolution using Planar-NN is first selected as below. Starting from the head gene (gene with an index 1), the operator searches the following genes if the genes pair satisfies one of the following cases.

(i) The genes pair forms a line which is parallel to one of the inertial frame axes.

(ii) The genes pair forms a plane which is parallel to the plane formed by any two of the inertial frame axes.

From Figure 18, the gene with an index 2 is the first observed gene which satisfies the first condition. Therefore, it is extracted and adjoined to the current gene. After that, the extracted gene becomes the current gene and the selection process is repeated until there is no genes left for extraction. If there is no genes matching the above conditions, the first unselected gene which follows the current gene is extracted. From the remaining genes, the second extracted gene is the one with an index 3 since the points pair with gene indexes 2 and 3 satisfies the second condition. By repeating the the extraction and adjoining processes, the final offspring is obtained.

The design of Planar-NN is a greedy search for an optimal solution. To avoid a local optimal solution, three conventional genetic operators are involved in the evolution process. They are (a) genes flip, (b) genes swap, and (c) genes slide. For the working principle of these conventional operators, it is illustrated in [24] and it is not repeated here.

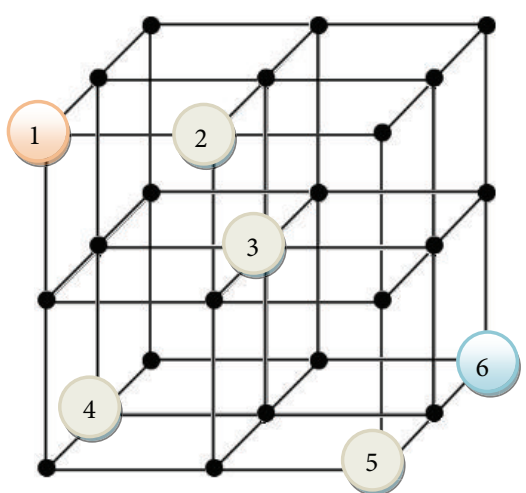

Before \begin{tabular}{|l|l|l|l|l|l|}
\hline 1 & 3 & 2 & 5 & 4 & 6 \\
\hline
\end{tabular}

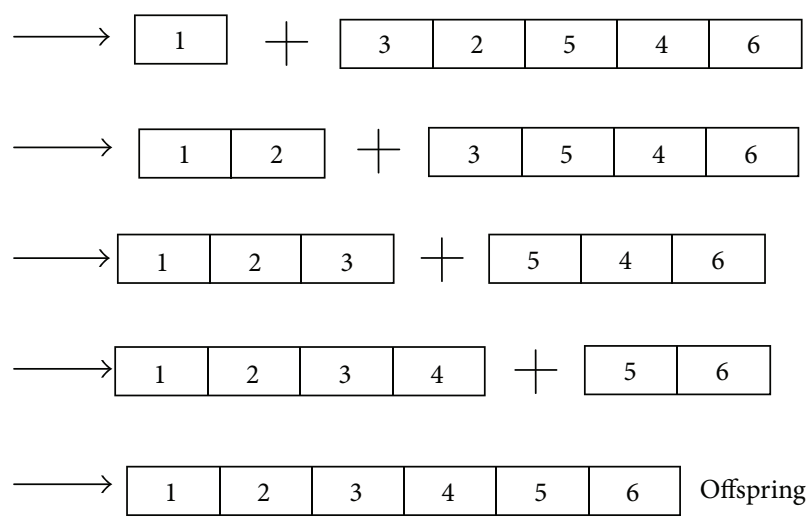

FIgURE 18: The idea of the proposed operator, Planar-NN.

TABLE 4: The key parameters for the simulations.

\begin{tabular}{lc}
\hline Robot initial position & $(4,29,4)$ \\
Robot initial head vector & $(1,0,0)$ \\
Robot initial normal vector & $(0,0,1)$ \\
Robot final position & $(31,26,25)$ \\
Maximum number of generation & 1000 \\
Number of experiments & 5 \\
\hline
\end{tabular}

5.4. Simulation Study and Discussion. In this section, the performance of the proposed algorithm is evaluated by simulating a path planning problem on a structural frame. To evaluate the algorithm under different aspects, different simulations are performed. Forty points are first randomly generated on a cubic frame structure and the edge length is 40 meters. It is divided into many smaller cells each with 1 meter long $(0 \leq x, y, z \leq 40)$. The genetic operators involved in the evolution process are (a) genes flip, (b) genes swap, (c) genes slide, and (d) Planar-NN. The probabilities of selecting them are equal during the evolution process and the maximum number of generation is 1000. Table 4 lists some of the key parameters for the simulations.

The first simulation aims to show the effect of different population sizes on the final solution path. The conventional 
TABLE 5: A comparison of convergent rate in the first 400 generations.

\begin{tabular}{lccccr}
\hline & \multicolumn{3}{c}{ Fitness at $n$th generation } \\
& 0 th & 100 th & 200 th & 300 th & 237.54 \\
\hline Conventional & 314.78 & 254.57 & 241.46 & 234.42 \\
Proposed & 314.78 & 237.49 & 235.23 & 232.55 \\
\hline
\end{tabular}

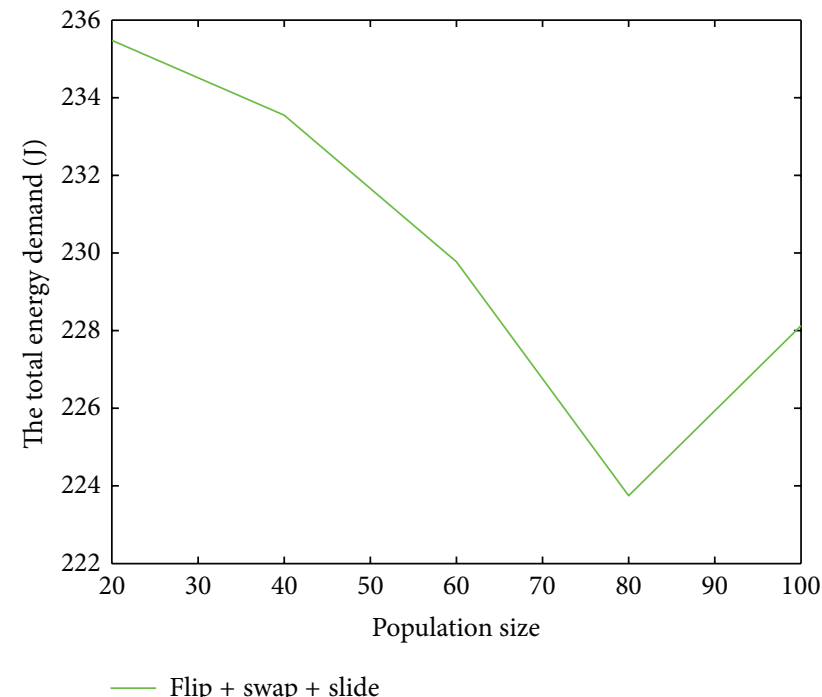

FIGURE 19: The total energy demand of the final solution with different population sizes.

genetic operators are utilized during the genetic evolution. At the same time, the maximum population size is varied. The simulation is repeated five times and the mean total energy demand of the best solution path is shown in Figure 19.

In general, the total energy demand of the best solution path searched by the algorithm decreases when the population size increases. It is reasonable because more chromosomes are involved in searching the global optimal path. From the simulation results, the best population size is selected as 80 .

In the second simulation, the proposed genetic operator, Planar-NN, is utilized to search a solution path and the result is compared with the results of using conventional genetic operators. The simulation is repeated 10 times and the mean of the ten simulation results is shown in Figure 20.

From Figure 20, the overall convergent rate is significantly enhanced when the proposed genetic operator is involved in the evolution process. Also, the proposed algorithm can search a solution path with a lower total energy demand. Table 5 gives a comparison of the convergent rate between the use of conventional operators alone and the hybrid use of conventional operators and Planar-NN.

When Plannar-NN is involved for genetic evolution, the total energy demand of a path represented by the current best chromosome is decreased below 255 joule in the first 100 generation. Also, the evolution process keeps improving the fitness of the current fittest chromosome and the result is closed to the optimal solution after 400 generations with

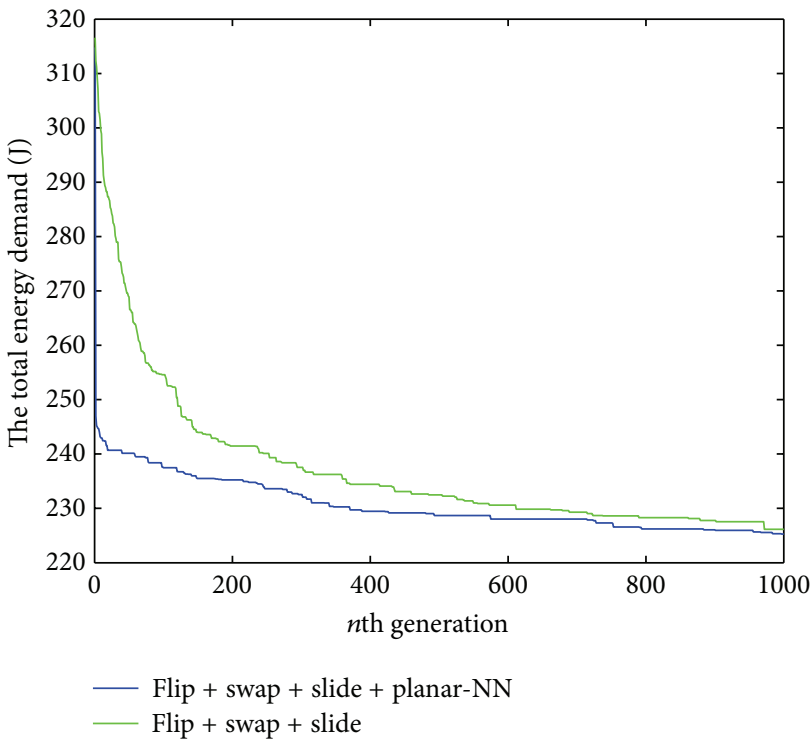

FIGURE 20: The effect of different genetic operators on the best path given by the proposed algorithm.

a variation within 11 joule. To save the computation time, the evolution process can be terminated if the total energy demand of the current best path is satisfied.

To explain these, the nature of the Planar-NN and the hybrid use of genetic operators are considered. The design of Planar-NN is a greedy local search. It groups the genes with a planar or linear relationships. Therefore, the use of Planar-NN can result in a solution path with segments of planar or linear check points. Also, the hybrid use of conventional genetic operators can maintain the concepts of natural evolution in GA and so local optimums can be avoided. Hence, the proposed algorithm can facilitate the search of a minimum energy demand path. Also, it verifies that the composition of motion primitives in a motion path is also important in energy demand minimization. Based on the simulation results, the total energy demand of the best solution path given by the proposed algorithm is 218.79 joule and the corresponding solution path is displayed in Figure 21. The green lines represent the edge of the frame structure and the blue points indicate the randomly generated check points. The initial and final robot positions are marked with black and pink dots, respectively.

\section{Conclusion and Future Work}

In this paper, we propose the design of new gaits for space manipulators with a lower energy demand which is the major 


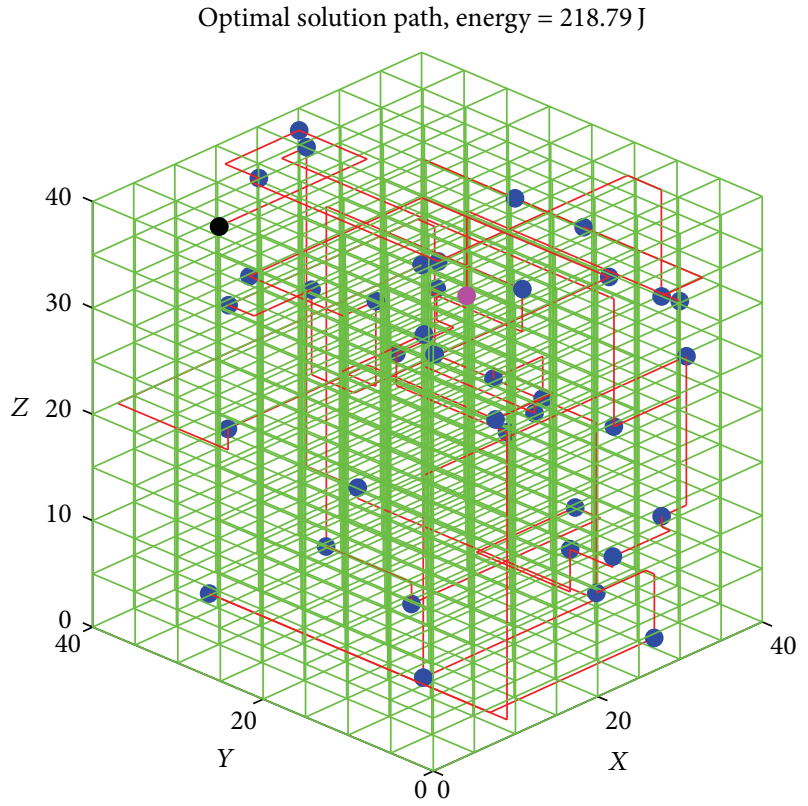

FIGURE 21: The best path given by the proposed algorithm.

contribution of this research. Based on the system dynamic model, different gaits are simulated and the corresponding energy demand is analyzed. Results show that the proposed gaits require a lower energy demand when compared with conventional gaits. With the use of the proposed gaits, a minimum energy demand-based path planning algorithm is also developed. Different from traditional approaches, the proposed algorithm considers the composition of motion primitives in a path for the energy demand minimization. Based on the framework of conventional GA, we adopted the concept of genetic modification to design a new genetic operator, Planar-NN. Based on the simulation results, it benefits the final solution in terms of fast convergent rate and lower energy demand. Also, it verifies that the composition of motion primitives in a path is also important in energy demand minimization. Although this paper assumes a space station as a cubic truss structure which is different from the existing space station such as International Space Station (ISS), the geometry of the cubic truss can be modified and the concept of nonfeasible edges can be introduced by highly increasing the energy demand of those edges. For the future development, the proposed algorithm will be able to handle uncertainties such as infeasible path and obstacle avoidance.

\section{References}

[1] P. Acquatella, "Development of automation \& robotics in space exploration," Journal of Robotics and Autonomous Systems, vol. 10, no. 106, 2008.

[2] MacDonald Dettwiler Space and Advanced Robotics, "Canadarm-the SRMS technical details," http://www.iee.ca/ millennium/canadarm/canadarm_technical.html.

[3] C. Sallaberger, "Canadian space robotic activities," Acta Astronautica, vol. 41, no. 4-10, pp. 239-246, 1997.
[4] K. Yoshida, "ETS-VII flight experiments for space robot dynamics and control-theories on laboratory test beds ten years ago, now in orbit," in Experimental Robotics VII, LNCIS, D. Rus and S. Singh, Eds., pp. 209-218, Springer, Berlin, Germany, 2001.

[5] N. Sato and Y. Wakabayashi, "JEMRMS design features and topics from testing," in Proceedings of the 6th International Symposium on Artificial Intelligence and Robotics \& Automation in Space (i-SAIRAS '01), Canadian Space Agency, St-Hubert, Quebec, Canada, 2001.

[6] R. Boumans and C. Heemskerk, "The European Robotic Arm for the International Space Station," Robotics and Autonomous Systems, vol. 23, no. 1-2, pp. 17-27, 1998.

[7] A. Kauderer, "NASA-Canadarm2 and the Mobile Servicing System," http://www.nasa.gov/mission_pages/station/structure/elements/mss.html.

[8] M. Vona, C. Detweiler, and D. Rus, "Shady: robust truss climbing with mechanical compliances," in Proceedings of the International Symposium on Experimental Robotics, 2006.

[9] Y. Yoon and D. Rus, "Shady3D: a robot that climbs 3D trusses," in Proceedings of IEEE International Conference on Robotics and Automation (ICRA '07), pp. 4071-4076, April 2007.

[10] M. Bonani, S. Magnenat, P. Retornaz, and F. Mondada, "The handbot, a robot design for simultaneous climbing and manipulation," in Proceedings of the 2nd International Conference on Intelligent Robotics and Applications, pp. 11-22, 2009.

[11] W. K. Chung, J. Li, Y. Chen, and Y. Xu, "A novel design of movable gripper for non-enclosable truss climbing," in Proceedings of IEEE International Conference on Robotics and Automation, Shanghai, China, May 2011.

[12] W. K. Chung and Y. Xu, "A novel frame climbing robot: frambot," in Proceedings of IEEE International Conference on Robotics and Biomimetics, pp. 2559-2566, Phuket, Thailand, 2011.

[13] J. A. Fernandez, J. Gonzalez, L. Mandow, and J. L. Pérez-de-laCruz, "Mobile robot path planning: a multicriteria approach," Engineering Applications of Artificial Intelligence, vol. 12, no. 4, pp. 543-554, 1999.

[14] M. Gemeinder and M. Gerke, "GA-based search for paths with minimum energy consumption for mobile robot systems," in Proceedings of IEEE International Conference on 7th Fuzzy Days on Computational Intelligence, Theory and Applications, vol. 2206, pp. 599-607, 2001.

[15] T. Wang, B. Wang, H. Wei, Y. Cao, M. Wang, and Z. Shao, "Staying-alive and energy-efficient path planning for mobile robots," in Proceedings of IEEE International Conference on American Control Conference, pp. 868-873, June 2008.

[16] C. C. Ooi and C. Schindelhauer, "Minimal energy path planning for wireless robots," Mobile Networks and Applications, vol. 14, no. 3, pp. 309-321, 2009.

[17] S. Liu and D. Sun, "Optimal motion planning of a mobile robot with minimum energy consumption," in Proceedings of IEEE International Conference on Advanced Intelligent Mechatronics (AIM '11), pp. 43-48, 2011.

[18] C. Balaguer, A. Giménez, J. M. Pastor, V. M. Padrón, and M. Abderrahim, "Climbing autonomous robot for inspection applications in 3D complex environments," Robotica, vol. 18, no. 3, pp. 287-297, 2000.

[19] C. Balaguer, V. M. Padron, A. Gimenez, J. M. Pastor, and M. Abderrahim, "Path planning strategy of autonomous climbing robot for inspection applications in construction," in Proceedings of the International Symposium on Automation \& Robotics in Construction, pp. 347-352, 1999. 
[20] Y. Guan, L. Jiang, X. Zhang, and H. Zhang, "Climbing gaits of a modular biped climbing robot," in Proceedings of IEEE/ASME International Conference on Advanced Intelligent Mechatronics (AIM '09), pp. 532-537, July 2009.

[21] Y. Xu, Space Robotics: Dynamics and Control, Kluwer Academic Publishers, 1992.

[22] Y. Xu, "Measure of dynamic coupling of space robot systems," in Proceedings of IEEE International Conference on Robotics and Automation, pp. 615-620, May 1993.

[23] M. Shibli, "Unified modeling approach of kinematics, dynamics and control of a free-flying space robot interacting with a target satellite," Journal of Intelligent Control and Automation, vol. 2, pp. 8-23, 2011.

[24] W. K. Chung and Y. Xu, "A generalized 3-D path planning method for robots using Genetic Algorithm with an adaptive evolution process," in Proceedings of the 8th World Congress on Intelligent Control and Automation (WCICA '10), pp. 1354-1360, Jinan, China, July 2010. 

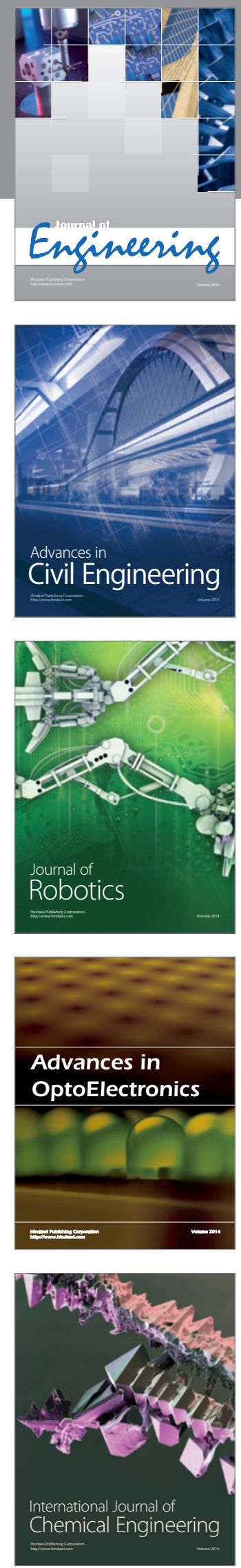

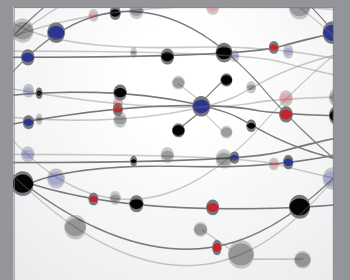

The Scientific World Journal
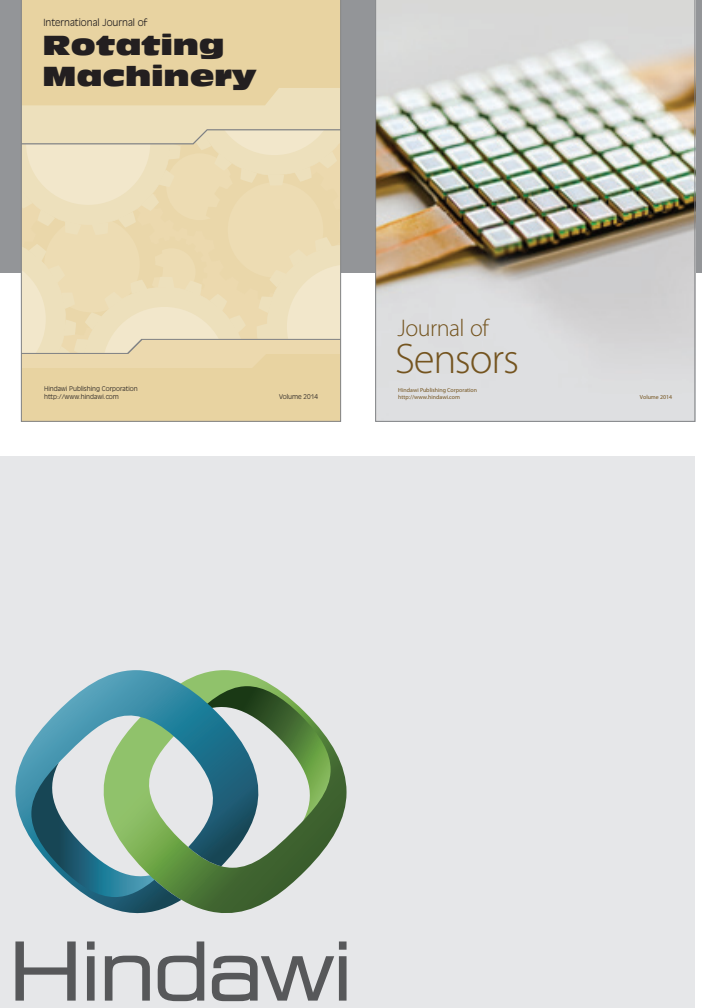

Submit your manuscripts at http://www.hindawi.com
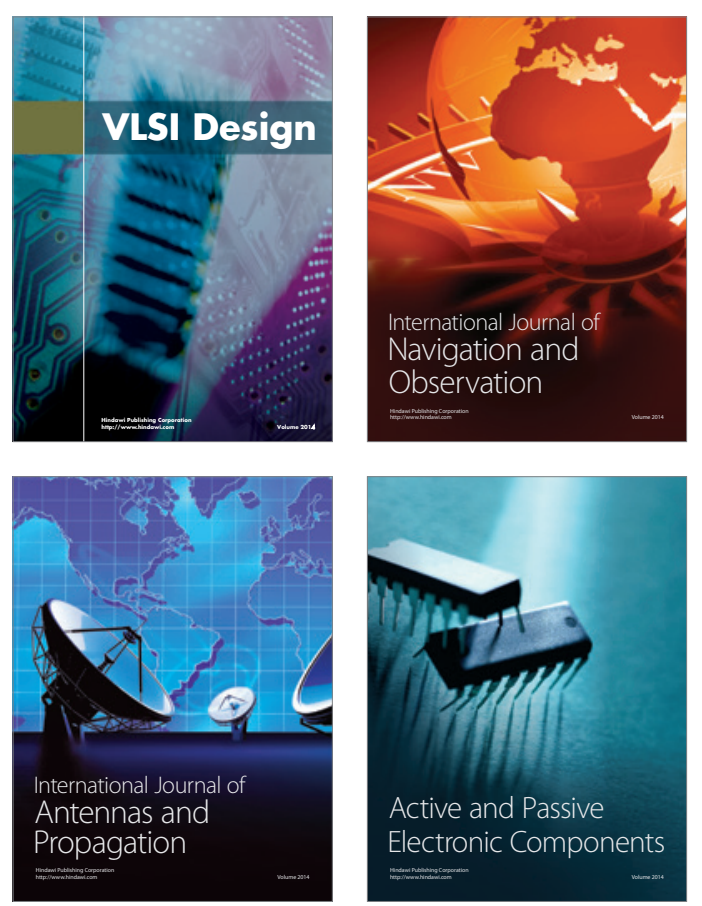
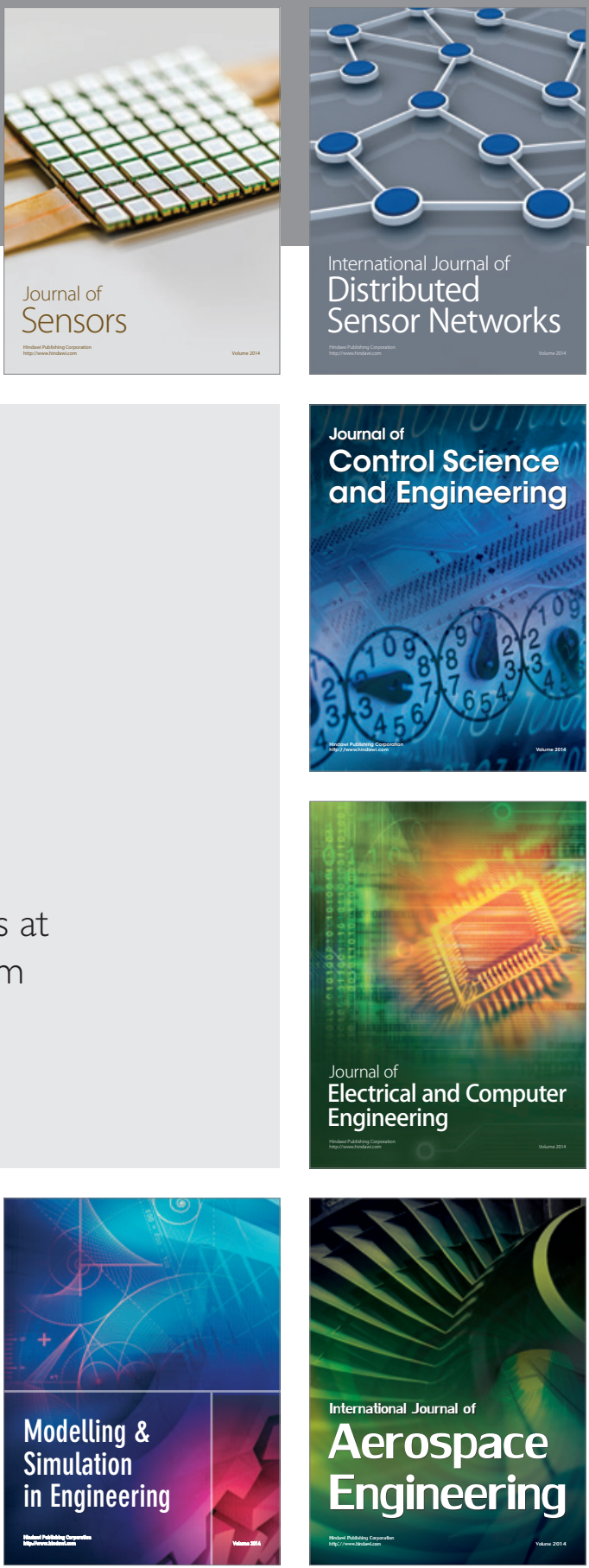

Journal of

Control Science

and Engineering
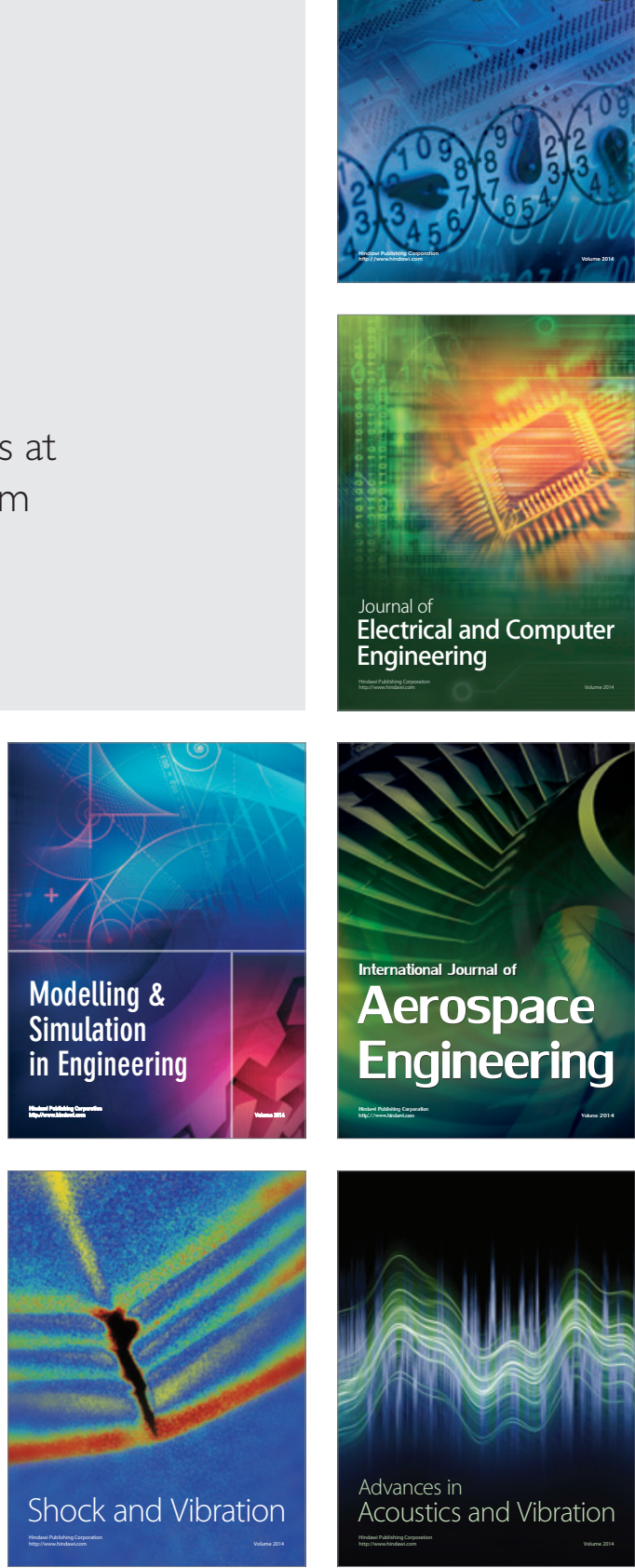